\title{
Emerging Technology Update
}

\section{Intravascular Photoacoustic Imaging of Vulnerable Atherosclerotic Plaque}

\author{
Min Wu, ${ }^{1}$ Antonius FW van der Steen, ${ }^{1,2,3,4}$ Evelyn Regar $^{5}$ and Gijs van Soest ${ }^{1}$ \\ 1. Department of Biomedical Engineering, Thorax Centre, Erasmus Medical Center, Rotterdam, The Netherlands; 2. Interuniversity Cardiology Institute \\ of The Netherlands, Netherlands Heart Institute, Utrecht, The Netherlands; 3. Department of Imaging Science and Technology, Delft University \\ of Technology, Delft, The Netherlands; 4. Shenzhen Institutes of Advanced Technology, Chinese Academy of Sciences, Shenzhen, China; \\ 5. Department of interventional cardiology, Thorax Center, Erasmus Medical Center, Rotterdam, The Netherlands
}

\begin{abstract}
The identification of vulnerable atherosclerotic plaques in the coronary arteries is emerging as an important tool for guiding atherosclerosis diagnosis and interventions. Assessment of plaque vulnerability requires knowledge of both the structure and composition of the plaque. Intravascular photoacoustic (IVPA) imaging is able to show the morphology and composition of atherosclerotic plaque. With imminent improvements in IVPA imaging, it is becoming possible to assess human coronary artery disease in vivo. Although some challenges remain, IVPA imaging is on its way to being a powerful tool for visualising coronary atherosclerotic features that have been specifically associated with plaque vulnerability and clinical syndromes, and thus such imaging might become valuable for clinical risk assessment in the catheterisation laboratory.
\end{abstract}

\section{Keywords}

Vulnerable plaque, intravascular photoacoustic imaging, atherosclerosis, intravascular imaging, coronary artery disease

Disclosure: The authors have no conflicts of interest to declare.

Submitted: 18 April 2016 Accepted: 8 September 2016 Citation: Interventional Cardiology Review 2016;11(2):120-3 DOI: 10.15420/icr.2016:13:3

Correspondence: Gijs van Soest, Department of Biomedical Engineering, Thorax Center, Erasmus University Medical Center, PO Box 2040, 3000 CA Rotterdam,

The Netherlands. E: g.vansoest@erasmusmc.nl

Vulnerable atherosclerotic plaque comprises a lipid-rich necrotic core, covered by a thin fibrous cap, that is weakened by macrophage infiltration. ${ }^{1}$ Plaques that have this morphology and composition have a greater probability of being associated with acute coronary syndromes in clinical studies. ${ }^{2,3}$ Coronary artery disease is most commonly triggered by the rupture of vulnerable plaque and thrombosis. ${ }^{4}$ The identification of vulnerable plaque is emerging as an important element in coronary artery disease diagnosis and treatment.

In the past three decades, several imaging modalities have been employed to identify vulnerable plaque. Coronary angiography is the oldest of these modalities and can only visualise the artery lumen, not the artery wall and atherosclerotic lesions. Moreover, angiography creates images of a three-dimensional moving object that are presented in a two-dimensional plane. Intravascular, catheter-based imaging methods can be safely used in a routine catheterisation laboratory population ${ }^{5}$ and offer direct visualisation of the artery wall. Intravascular ultrasound (IVUS) imaging has been used in routine clinical practice for more than 20 years. Due to the limited acoustic contrast between soft tissues, the conventional grayscale IVUS image is not sensitive enough to differentiate plaque composition, except for the presence of calcium. ${ }^{6.7}$ While the application of an advanced analysis algorithm to IVUS images - virtual histology IVUS - has improved plaque composition characterisation, ${ }^{8}$ no data yet support its accuracy in the detection of lipid-rich necrotic plaque core. ${ }^{9-11}$ IVUS palpography is a technology used to assess local mechanical plaque properties based on tissue deformation caused by intraluminal pressure..$^{12}$ It can only sense the stiffness of the tissue, however, and cannot directly image the tissue, and it only assesses the first $450 \mu \mathrm{m}$ of the arterial wall. ${ }^{12}$ Light-based intravascular optical coherence tomography (IVOCT) creates high-resolution images (approximately $15 \mu \mathrm{m})$ with backscattered light from the tissue ${ }^{13}$ and can image plaque components such as calcium, lipid and thrombus. IVOCT currently relies on qualitative image interpretation to distinguish these features. ${ }^{14,15}$ The depth to which IVOCT penetrates is only $1-2 \mathrm{~mm}$ into the atherosclerotic tissue, which means it often cannot see the extent of the plaque up to the adventitia. Near-infrared spectroscopy (NIRS) is another light-based imaging modality for the detection of lipid-rich necrotic core in plaque. ${ }^{16}$ It provides a probability for the presence of coronary lipid based on the spectral analysis of backscattered light; ${ }^{17}$ however, NIRS cannot identify the amount and location of lipids, which may be important in risk assessment. ${ }^{18}$

Several other innovative optical modalities have been proposed recently ${ }^{19-22}$ that are currently being evaluated in clinical trials. The ability of these technologies to provide reliable, spatially-precise, quantitative imaging of plaque lipid content is yet to be determined. In this paper, a newer modality is reviewed: intravascular photoacoustic (IVPA) imaging. This is an optical-acoustic hybrid imaging modality. IVPA is currently being developed to show both the morphology and chemical composition of the artery wall with a good imaging depth and resolution. This technology takes advantage of the unique 
optical absorption contrast between different tissues and reasonably large penetration depth owing to the low acoustic attenuation in soft tissues. ${ }^{23,24}$ Published experimental data suggest it may be a strong contender for detailed plaque characterisation and the identification of plaque at increased risk of causing clinical sequelae.

\section{The Principles of IVPA Imaging of Atherosclerotic Plaque}

IVPA imaging requires short laser pulses, lasting a few nanoseconds, to be applied to irradiate the artery wall. Absorption of the light converts the optical energy into heat in the tissue and causes thermoelastic expansion, leading to a transient pressure rise (the laser pulse duration is shorter than both the stress relaxation and the thermal diffusion times). Figure 1 illustrates the principles involved in IVPA imaging.

The specific optical absorption spectra of lipids and other pure substances can be related to the identification of plaque. ${ }^{25}$ Various biomarkers present in plaques have been explored for their usefulness in IVPA imaging, with lipid being the most frequently used. IVPA imaging of plaque lipids can be performed at around $1.2 \mu \mathrm{m}$ or $1.72 \mu \mathrm{m}$ due to the high lipid-specific absorption contrast with relatively high resolution $(\sim 50 \mu \mathrm{m})$ and reasonable penetration depth. ${ }^{26}$ At $1.2 \mu \mathrm{m}$ the laser's maximal permissible exposure limit is $20 \mathrm{~mJ} / \mathrm{cm}^{2}$ and at $1.72 \mu \mathrm{m}$ it is $1 \mathrm{~J} / \mathrm{cm}^{2}$, according to the American National Standards Institute laser safety standard. ${ }^{27,28}$ NIRS also makes use of lipid to identify potentially vulnerable plaque, but there is no depth resolution. The capability of IVPA to produce images of atherosclerotic plaque lipids has been demonstrated extensively with ex vivo tissues and in vivo animal models. ${ }^{26,27,29-33}$

Macrophages are an important cell type related to the progression of atherosclerosis ${ }^{34}$ and the specific imaging of plaque macrophage content is a potentially interesting feature when identifying vulnerable plaque. ${ }^{36}$ Macrophages do not exhibit a strong intrinsic contrast in the way lipids do, therefore exogenous imaging contrast agents with high optical absorption are applied in order to visualise them using IVPA imaging. ${ }^{36,37}$ Furthermore, given that the activity of the enzyme matrix metalloproteinase (MMP) is an indicator of plaque instability, the localisation of MMP activity with an MMP-sensitive activatable probe in vulnerable plaques in human carotid specimens has been achieved using photoacoustic imaging. ${ }^{38}$ More recently, an ApoE- mouse in vivo model injected with a photoacoustic ICG@PEG-Ag2S nanoprobe was successful in imaging plaque. ${ }^{39}$

\section{Developing IVPA Imaging for Clinical Use}

Encouraged by the capability of IVPA to create images of vulnerable atherosclerotic plaque, an intensive research effort is on-going to incorporate IVPA imaging into clinical applications.

\section{Miniaturising the IVPA Catheter}

A miniature, and highly sensitive, catheter for IVPA imaging is an essential element if IVPA imaging technology is to enter common use. As IVPA is an intrinsically multimodal imaging technique, the catheter's technical requirements are harder to meet than for single-modality probes like optical coherence tomography or IVUS catheters. A typical IVPA imaging catheter consists of an optical part for light delivery and a broadband ultrasound transducer. The optical part is usually a combination of an optical fibre and a mirror or a single-angle polished optical fibre.40 Two IVPA catheter designs are shown in Figure 2. The unit shown in Figure $2 A$ provides the
Figure 1: Illustration of the Intravascular Photoacoustic Imaging Principle

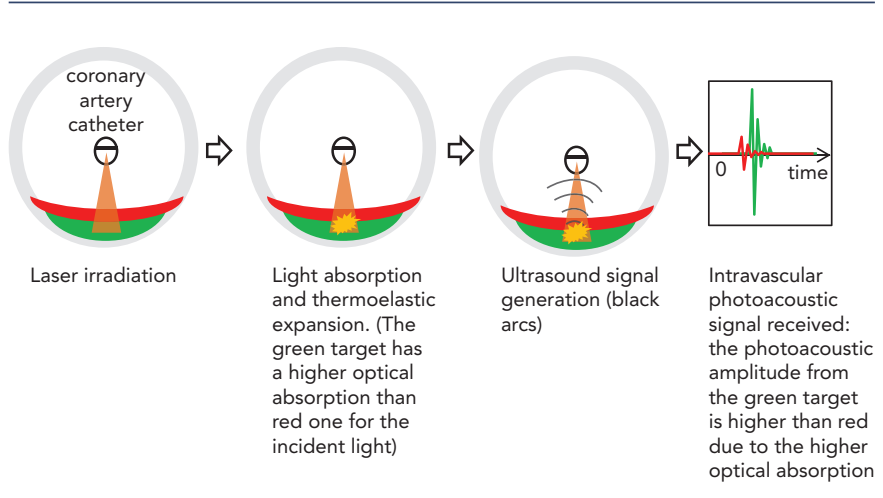

Figure 2: Different Intravascular Photoacoustic (IVPA) Catheter Designs
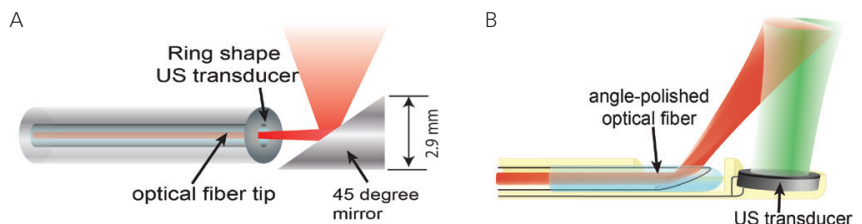

(A) A collinear IVPA catheter. (B) An IVPA catheter with a longitudinal offset between optical and acoustic beams, where red is the optical beam and green the ultrasound beam ladapted from wu et al., 2010 41 .

greatest reported sensitivity. Miniaturisation is challenging, however, and this unit is currently too large for intravascular application, having a diameter of $2.9 \mathrm{~mm} .{ }^{27.28}$ Another design possibility - one that is favoured for miniaturisation - incorporates an offset between the optical fibre and ultrasound transducer, either longitudinally $y^{41,42}$ (see Figure 2B) or laterally. ${ }^{43}$ The smallest IVPA catheter reported to date that uses this design has an outer diameter of $0.9 \mathrm{~mm}$, which is below the threshold of $1 \mathrm{~mm}$ desired for clinical translation into coronary arteries. ${ }^{44}$

\section{Efficient and Specific Plaque Identification}

Lipids are found in the plaque inside the artery wall, which is rich in cholesterol and cholesteryl esters, ${ }^{45,46}$ as well as in peri-adventitial fat tissue around the artery, which contains a mixture of fatty acids. ${ }^{47}$ The spectral contrast between plaque lipids and peri-adventitial fat makes it possible to differentiate between them using spectroscopic IVPA imaging (SIVPA). ${ }^{26}$ As a consequence of this, the presence and location of lipids within a plaque can be reliably and rapidly displayed during catheter pullback, using both cross-sectional and longitudinal data display. Performing multiple wavelength SIVPA imaging, however, has an impact on the complexity, cost andspeed of the imaging system. Minimisation of the number of wavelengths for the differentiation of different lipids or improving the efficiency for specific lipid differentiation is necessary for practical application. The possibility of specific plaque lipid imaging using sIVPA with limited number of wavelengths has been investigated (see Figure 3). ${ }^{26,48} \mathrm{It}$ is possible to achieve about $70 \%$ true positive and $15 \%$ false positive pixel identification of plaque lipids based on the relative difference between amplitudes of only two wavelengths (see Figure 4). ${ }^{49}$

High-speed IVPA Imaging of Atherosclerotic Plaque Despite successful plaque identification by IVPA imaging, the Iow imaging speed limits its application in practice. Lipid specificity remains limited by the single-wavelength operation and the average optical power remains quite high (up to $1 \mathrm{~W}$ ), which raises concerns about 
Figure 3: Lipid Identification in an Atherosclerotic Human Coronary Artery
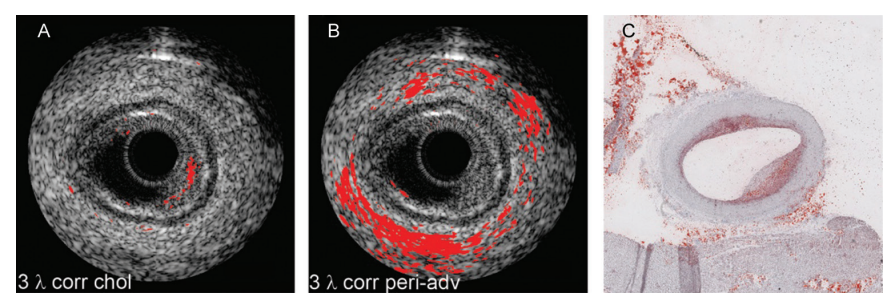

(A) Plaque lipid identification based on a three-wavelength correlation with the reference spectra of cholesterol in the $1.2 \mathrm{~mm}$ spectral range. (B) Peri-adventitial fat identification based on three-wavelength correlation with the reference spectra of human peri-adventitial fat tissue. (C) Histology result following Oil Red O staining. All lipid identifications have been laid over registered intravascular ultrasound images. Adapted from Jansen et al., 2013.48

thermal damage to the patient's artery and the potential hazard to the operator resulting from skin and eye exposure. With the introduction of higher pulse-rate laser systems (2 kHz), IVPA imaging speed has been increased from about 0.3 to 1 frame per second ${ }^{27,28,50}$ This represents a significant step forward, but is still not practical. More and faster IVPA imaging systems are, however, on the horizon. A fast multiplewavelength sIVPA imaging system with potentially $\sim 5$ frames per second has been developed that is capable of imaging at a low-energy pulse. ${ }^{44}$

\section{Discussion}

IVPA imaging shows great promise in detecting the morphology and composition (e.g. lipid deposition) of arterial lipid plaque burden. Owing to the extensive research on IVPA imaging, several important milestones have been passed, with the development of a miniature flexible catheter ( $<1 \mathrm{~mm}$ in diameter), high-efficiency specific imaging of vulnerable plaque composition and the development of a highspeed IVPA imaging system ( $\sim 5$ frames per second). Some challenges remain, however, in the further development of IVPA imaging.

Most current IVPA imaging systems require the artery to be cleared of blood by flushing for a better photoacoustic signal-to-noise ratio. It is in principle possible to perform IVPA imaging at $1.72 \mu \mathrm{m}$ without flushing, due to the similarity in the optical absorption property of blood and water-based tissues. Wang et al. performed the first in vivo IVPA imaging (at 1,720 nm) in a hypercholesterolemic rabbit model through blood. ${ }^{29}$ Further IVPA imaging through blood - and in and ex vivo human tests - must await the resolution of other clinical implementation issues described in this article. Although the imaging speed has been significantly improved, ${ }^{51-53}$ it is still not fast
Figure 4: Ex Vivo Lipid Differentiation Result of the Atherosclerotic Left Anterior Ascending Coronary Artery of an 80-year-old Man
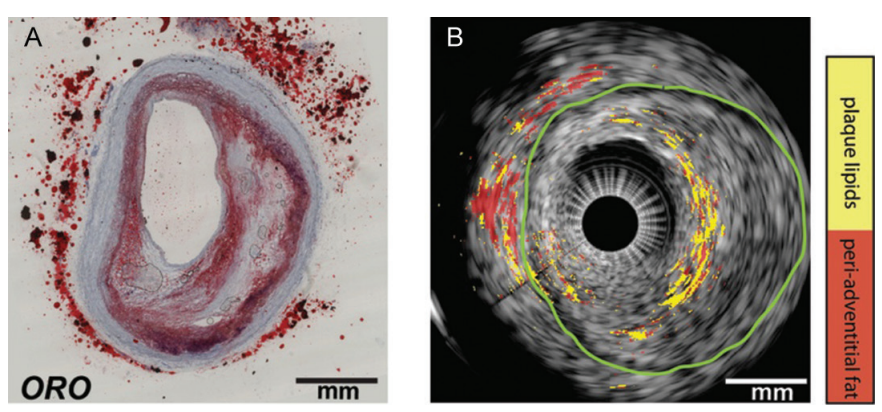

(A) Histology: Oil Red O (ORO) staining of the intravascular photoacoustic/intravascular ultrasound image in cross-section. The lipids are shown in red. (B) Lipid differentiation map overlaid on the co-registered intravascular ultrasound image of the coronary artery. The lipids in the plaques are yellow and lipids in the peri-adventitial tissue are red. The green contour indicates the external elastic lamina. Adapted from Wu et al., 2015.49

enough for clinical use. Recently, it was found that the sensitivity of IVPA catheters can be increased by an order of magnitude by matching the frequency response of the receiving transducer to the low-frequency range. ${ }^{54,55}$ This enables imaging with lower pulse energy, allowing for fast laser sources with moderate output power. Other challenges in adapting IVPA imaging to the clinical setting include the cost and robustness of the laser system and the choice of catheter sheath material, which needs to be transparent for ultrasound and infrared light. These topics being investigated in on-going engineering research.

\section{Conclusion}

IVPA imaging is unique in its biochemical specificity and can offer the clinician direct, validated visualisation of coronary plaque lipid. To many clinicians, the ideal tool for guiding atherosclerotic plaque diagnosis and interventions requires high resolution to characterise the thin cap, high sensitivity to detect lipids for plaque visualisation in the artery, and a reasonable depth of penetration for plaque burden assessment. To achieve this, the combination of IVOCT and IVPA appears to be a promising choice, but at the cost of integrating an extra optical coherence tomography laser system. However, the natural progression of plaque with vulnerable characteristics is complex and the development and application of clinical tools for its assessment is, likewise, challenging. To date, IVPA imaging remains a research tool; future advances in technology will determine to what degree it will be of use in clinical practice.
1. Schaar JA, Muller JE, Falk E, et al. Terminology for highrisk and vulnerable coronary artery plaques. Eur Heart 2004;25:1077-82. PMID: 15191780

2. Kini AS, Motoyama S, Vengrenyuk Y, et al. Multimodality intravascular imaging to predict periprocedural myocardi intravascular imaging to predict periprocedural myocardi infarction during percutaneous coronary intervention. JACC Cardiovasc Interv 2015;8:937-45. DC
j.jcin.2015.03.016; PMID: 26088511

3. Goldstein JA, Maini B, Dixon SR, et al. Detection of lipidcore plaques by intracoronary near-infrared spectroscopy core plaques by intracoronary near-infrared spectroscopy
identifies high risk of periprocedural myocardial infarction. identifies high risk of periprocedural myocardial inf
Circ Cardiovasc Interv 2011;4:429-37. DOI: 10.1161/ Circ Cardiovasc Interv 2011;4:429-37. DOI: 10.1161/ CIRCINTERVENTIONS.111.963264; PMID: 219723991.

World Health Organization. Cardiovascular diseases (CVDs). January 2015. Available at: http://Www.who.int/topics/ cardiovascular_diseases/en/ (accessed 9 September 2016)

van der Sijde JN, Karanasos A, van Ditzhuijzen NS, et al. Safety of optical coherence tomography in daily practice: Cardiovasc Imag 2016:jew037. DOI: http://dx.doi.org/10.1093/ Cardiovasc Imag 20
ehici/jew037

ehjci/jew037
Allen T,, Hall A, Dhillon AP, et al. Spectroscopic photoacoustic imaging of lipid-rich plaques in the human aorta in the 740 to $1400 \mathrm{~nm}$ wavelength range. J Biomed Opt 2012:17:061209-10. DOl: 10.1117/1.JBO 17.6.061209; PMID: 22734739

7. Choudhury RP, Fuster V, Fayad ZA. Molecular, cellular and functional imaging of atherothrombosis. Nat Rev Drug Discov 2004;3:913-25. PMID: 15520814

8. Nair A, Kuban BD, Tuzcu EM, et al. Coronary plaque Nair A, Kuban BD, Tuzcu EM, et al. Coronary plaque
classification with intravascular ultrasound radiofrequency data analysis. Circulation 2002;106:2200-6. PMID: 12390948

9. Thim T, Hagensen MK, Wallace-Bradley D, et al. Unreliable assessment of necrotic core by virtual histology assessment of necrotic core by vifual histology Circ Cardiovasc Imaging 2010;3:384-91. DOI: 10.1161/ CIRCIMAGING.109.919357; PMID: 20460496

10. Shin ES, Garcia-Garcia HM, Ligthart JM, et al. In vivo findings of tissue characteristics using iMap IVUS and virtual of tissue characteristics using iMap IVUS and virtual
histology IVUS. Eurolntervention 2011;6:1017-9. DOI: 10.4244/ histology IVUS. Eurolntervention
EIJV618A175: PMID: 21330252

11. Puri R, Worthley MI, Nicholls SJ. Intravascular imaging of vulnerable coronary plaque: current and future concepts. Nat Rev Cardiol 2011;8:131-9. DOI: 10.1038/nrcardio.2010.210; PMID: 21263456
12. Schaar JA, van der Steen AF, Mastik F, et al. Intravascular palpography for vulnerable plaque assessment I Am Coll Cardiol 2006;47:C86-91. PMID: 16631515

13. Tearney GJ, Regar E, Akasaka T, et al; International Working Group for Intravascular Optical Coherence Tomography Group for Intravascular Optical Coherence Tomog
(IWG-IVOCT). Consensus standards for acquisition, measurement, and reporting of intravascular optical coherence measurement, and reporting of intravascular optical coherence
tomography studies: a report from the International Working Group for Intravascular optical coherence Tomography Standardization and Validation. J Am Coll Cardiol 2012;59:1058-72. DOI: 10.1016/j.jacc.2011.09.079; PMID: 22421299

14. Jansen $K$, van Soest $G$, van der Steen AFW. Intravascular photoacoustic imaging: A new tool for vulnerable plaque photucoustic inaging. A new tool for vilnerable plaque 10.1016/j.ultrasmedbio.2014.01.008; PMID: 24631379

15. van Soest G, Goderie T, Regar E, et al. Atherosclerotic tissue characterization in vivo by optical coherence tomography characterization in vivo by optical coherence tomography
attenuation imaging. J Biomed Opt 2010;15:011105. DOI: 10.1117/1.3280271: PMID: 20210431

16. Gardner CM, Tan H, Hull EL, et al. Detection of lipid core coronary plaques in autopsy specimens with a novel catheter-based near-infrared spectroscopy system.
JACC Cardiovasc Imaging 2008;1:638-48. DOI: 10.1016/j. 
jcmg.2008.06.001; PMID: 19356494

17. Waxman $S$, Dixon SR, LAllier $P$, et al. In vivo validation of a catheter-based near-infrared spectroscopy system for detection of lipid core coronary plaques: initial results of the SPECTACL study. J Am Coll Cardiol Img 2009;2:858-68. DOI: 10.1016/.j.jmg.2009.05.001; PMID: 19608137

18. Niccoli G, Liuzzo G, Montone RA, et al. Advances in mechanisms, imaging and management of the unstable plaque. Atherosclerosis 2014;233:467-77. DOI: 10.1016/ j.atherosclerosis.2014.01.036; PMID: 24530781

19. Vinegoni C, Botnaru I, Aikawa E, et al. Indocyanine green enables near-infrared fluorescence imaging of lipid-rich, inflamed atherosclerotic plaques. Sci Trans/ Med 2011;3: 84ra45. DOI: 10.1126/scitransImed.3001577; PMID: 21613624

20. Ughi GJ, Wang H, Gerbaud $\mathrm{E}$, et al. Clinical characterization of coronary atherosclerosis with dual-modality OCT and nearinfrared autofluorescence imaging .JACC Cardiovasc Imaging 2016; DOl: 10.1016/.j.jmg. 2015.11.020; PMID: 26971006 PMCID: epub ahead of press

21. Nadkarni SK, Bouma BE, Helg T, et al. Characterization of atherosclerotic plaques by laser speckle imaging. Circulation 2005;112:885-92. DOI: 10.1161/ CIRCULATIONAHA.104.520098; PMID: 16061738

22. van Soest $G$, van der Steen AFW, Regar E. Autofluorescence: a new NIR on the block. JACC Cardiovasc Imaging 2016;
DOI:10.1016/j.jcmg.2015.12.011; PMID: 26971002: epub ahead of press

23. Jansen $K$, van Soest $G$, van der Steen $T$. Photoacoustic imaging of coronary arteries: current status and potential clinical application. In: Arampatzis C, McFadden EP, Michalis LK, Virmani R, Serruys PW (eds). Coronary Atherosclerosis: Current Management and Treatment. First edn. London: Inform

24. Schoenhagen P, Vince DG. Intravascular photoacoustic tomography of coronary atherosclerosis riding the waves of light and sound. I Am Coll Cardiol 2014;64:391-3 DOI: 10.1016/j.jacc.2014.05.018; PMID: 25060375

25. Fleming CP, Eckert J, Halpern EF, et al. Depth resolved detection of lipid using spectroscopic optical coherence tomography. Biomed Opt Express 2013;4:1269-84. DOI: 10.1364/BOE.4.001269; PMID: 24009991

26. Jansen $\mathrm{K}$, Wu M, van der Steen AF, et al. Photoacoustic imaging of human coronary atherosclerosis in two spectral bands. Photoacoustics 2014;2:12-20. DOI: 10.1016/j.pacs.2013.11.003 27. Wang $\mathrm{P}, \mathrm{Ma}$ T, Slipchenko MN, et al. High-speed Intravascula
photoacoustic imaging of lipid-laden atherosclerotic plaque enabled by a 2-KHz barium nitrite raman laser. Sci Rep 2014;4:6889. DOI: 10.1038/srep06889; PMID: 25366991

28. Hui J, Yu Q, Ma T, et al. High-speed intravascular photoacoustic imaging at $1.7 \mu \mathrm{m}$ with a KTP-based OPO. Biomed Opt Express 2015;6:4557-66. DOI: 10.1364/ BOE.6.004557; PMID: 26601018

29. Wang B, Karpiouk A, Yeager D, et al. Intravascular photoacoustic imaging of lipid in atherosclerotic plaques in the presence of luminal blood. Opt Lett 2012;37:1244-6. DOI: 10.1364/OL.37.001244; PMID: 22466209

30. Allen TJ, Hall A, Dhillon A, et al. (eds). Photoacoustic imaging of lipid rich plaques in human aorta. In: Oraevsky AA, Wang LV (eds) Photons Plus Ultrasound: Imaging and Sensing 2010. San Francisco, California: SPIE, 2010. DOI: $10.1117 / 12.842205$

31. Jansen K, van der Steen AFW, van Beusekom HMM, et al. Intravascular photoacoustic imaging of human coronary atherosclerosis. Opt Lett 2011;36:597-9. DOI: 10.1364/ OL.36.000597; PMID: 21368919

32. Wang B, Su JL, Amirian J, et al. Detection of lipid in atherosclerotic vessels using ultrasound-guided spectroscopic intravascular photoacoustic imaging opt Express 2010:18:4889-97. DOI: 10.1364/OE.18.004889: PMID: 20389501

33. Wang $\mathrm{P}$, Wang HW, Sturek $\mathrm{M}$, et al. Bond-selective imaging of deep tissue through the optical window between 1600 and $1850 \mathrm{~nm}$. B Biophotonics 2012:5.25-32. D01: 10.1002 jbio.201100102; PMID: 22125288

34. Hansson GK. Inflammation, atherosclerosis, and coronary artery disease. N Engl J Med 2005;352:1685-95. PMID: 15843671

35. Tearney GJ, Yabushita H, Houser SL, et al. Quantification of macrophage content in atherosclerotic plaques by optical macrophage content in atherosclerotic plaques by
coherence tomography. Circulation 2003;107:113-9. coherence tomogra

36. Wang B, Joshi P, Sapozhnikova V, et al. (eds). Intravascular photoacoustic imaging of macrophages using molecularly targeted gold nanoparticles. Proc. SPIE 7564, Photons Plus Ultrasound: Imaging and Sensing 2010 2010:75640A. DOl: 10.1117/128 841070

37. Wang $B$, Yantsen $E$, Larson $T$, et al. Plasmonic intravascular photoacoustic imaging for detection of macrophages in atherosclerotic plaques. Nano Lett 2009;9:2212-7. DOI: 10.1021/nl801852e; PMID: 18844426

38. Razansky D, Harlaar NJ, Hillebrands JL, et al. Multispectral optoacoustic tomography of matrix metalloproteinase activity in vulnerable human carotid plaques. Mol Imaging Biol 2012;14:277-85. DOI: 10.1007/s11307-011-0502-6.

39. Wu C, Zhang Y, Li Z, et al. A novel photoacoustic nanoprobe of ICG@ PEG-Ag 2 S for atherosclerosis targeting and imagin in vivo. Nanoscale 2016;8:12531-9. DOI: 10.1039/c6nro0060f; PMID: 26853187

40. Karpiouk AB, Wang B, Emelianov SY. Development of a catheter for combined intravascular ultrasound and photoacoustic imaging. Rev Sci Instrum 2010;81:014901. DOI: 10.1063/1.3274197; PMID: 20113121

41. Wu M, Jansen $\mathrm{K}$, Springeling $\mathrm{G}$, et al. Impact of device geometry on the imaging characteristics of an intravascular photoacoustic catheter. Appl Opt 2014;53:8131-9. DOI: 10.1364/AO.53.008131; PMID: 25607973

42. Jansen $\mathrm{K}$, Springeling $\mathrm{G}$, Lancee $\mathrm{C}$, et al. An intravascular photoacoustic imaging catheter. Presented at: IEEE
International Ultrasonics Symposium, San Diego, 11-14 October 2010

43. Li X, Wei W, Zhou Q, et al. Intravascular photoacoustic maging at 35 and $80 \mathrm{MHz}$. J Biomed Opt 2012;17:106005-1. DOI: 10.1117/1.JBO.17.10.106005; PMID: 23224004

44. Li Y, Gong X, Liu C, et al. High-speed intravascular spectroscopic photoacoustic imaging at $1000 \mathrm{~A}$-lines pe second with a 0.9-mm diameter catheter. J Biomed Opt 2015;20:065006. DOI: 10.1117/1.JBO.20.6.065006; PMID: 26098356

45. Lundberg B. Chemical composition and physical state of lipid deposits in atherosclerosis. Atherosclerosis 1985;56:93-110. PMID: 4026939

46. Stegemann C, Drozdov I, Shalhoub J, et al. Comparative lipidomics profiling of human atherosclerotic plaques. Circ Cardiovasc Genet 2011:4:232-42. DOI: 10.1161/ CIRCGENETICS. 110.959098; PMID: 21511877

47. Tsai CL, Chen JC, Wang WJ. Near-infrared absorption property of biological soft tissue constituents. J Med Biol Eng 2001;21:7-14

48. Jansen $\mathrm{K}$, Wu M, van der Steen AF, et al. Lipid detection in atherosclerotic human coronaries by spectroscopic intravascular photoacoustic imaging. Opt Express 2013;21:21472-84. DOI: 10.1364/OE.21.021472; PMID: 24104022

49. Wu M, Jansen $K$, van der Steen $A F$, et al. Specific imaging of atherosclerotic plaque lipids with two-wavelength ascular photoacoustics. Biomed or 3276-86. DOI: 10.1364/BOE.6.003276; PMID: 264175

50. Piao Z, Ma T, Li $\rfloor$, et al. High speed intravascular photoacoustic imaging with fast optical parametric oscillato aser at 1.7 um. Appl Phys Lett 2015:107:083701. PMID: 26339072

51. Sethuraman S, Aglyamov SR, Amirian JH, et al. Intravascular photoacoustic imaging using an IVUS imaging catheter. IEE Trans Ultrason Ferroelectr Freq Control 2007;54(5):978-86. PMID: 17523562

52. Sethuraman S, Amirian JH, Litovsky SH, et al. Ex vivo characterization of atherosclerosis using intravascula photoacoustic imaging. Opt Express 2007;15:16657-66. PMID: 19550952

53. Sethuraman S, Amirian JH, Litovsky SH, et al. Spectroscopic intravascular photoacoustic imaging to differentiate atherosclerotic plaques. Opt Express 2008;16:3362-7 PMID: 18542427

54. Daeichin $\mathrm{V}$, Wu M, de Jong $\mathrm{N}$, et al. Frequency analysis of the photoacoustic signal generated by coronary atherosclerotic plaque. Ultrasound Med Biol 2016;42:2017-25. DOI: 10.1016 / j.ultrasmedbio.2016.03.015; PMID: 27181689

55. Daeichin V, Chen C, Ding Q, et al. A broadband polyvinylidene difluoride-based hydrophone with integrated readout circuit for intravascular photoacoustic imaging. Ultrasound Med Biol 2016;42:1239-43. DOI: 10.1016/ .ultrasmedbio.2015.12.016; PMID: 26856788 